A nother amplification or wave growth process which has received attention recently is the transverse resonance instability. In this instability, which leads to the generation and growth of VLF waves in the magnetosphere, the high energy tail of the trapped particle distribution is unstable if the pitch angle distribution is anisotropic. The process is thought to explain ELF and VLF hiss at medium latitudes. Kennel and Petschek ( $J$. geophys. Res., 72, 1; 1966) originally showed that particles with energies in excess of the magnetic energy for particles $\left(B^{2} / 8 \pi N\right.$, where $B$ is the magnetic field strength and $N$ is the ambient plasma density) would be unstable to wave growth and that the instability would lead to panticle precipitation and a limit to the fluxes of particles trapped in the magnetosphere. This limit to the numbers of trapped particles was considered important and in agreement with satellite observations. The existence of this limit is now questioned by Etcheto and her colleagues (J. geophys. Res., 78, 8150; 1973) who have established a self-consistent solution to the equations governing the wave growth and particle diffusion. This solution suggests that there is strictly no 'limiting flux' and that the equilibrium flux of trapped particles depends on the rate of injection of particles into the trapping region of the magnetosphere.

The amplifying and wave growth processes are clearly of increasing importance in this field for they contribute in different ways to both the strength of the transmitted whistler mode signal and the background noise which interferes with its reception.

A.R.W.H.

\section{Aspirin, Boston and Little Rock}

\section{from a Correspondent}

IT may be asserted with some confidence that the genetically determined haemoglobin variants provide the most impressive roll-call of place names from all over the world, from Saskatoon to Sydney, and from Toronto to Tongariki. The appearance of aspirin in the title of this column, however, calls initial attention to a paper which appears to dispose of yet another proposed therapy for sickle cell anaemia. Starting from the established transfer, both in vitro and in vivo, of the acetyl group of aspirin to an $\varepsilon-\mathrm{NH}_{2}$ group of lysine of serum albumin, Klotz and Tamm in 1973 had reported a similar transfer to haemoglobin, accompanied by a substantial increase in oxygen affinity. It was considered that this shift might be due to acetylation of the $\mathrm{NH}_{2}$ terminal valines; a similar shift is observed following their carbamyla- tion by cyanate, which is being intensively studied as a possible drug for the inhibition of sickling of sickle-cell anaemia red cells in vivo (see Nature new Biol., 242, 33; 1973).

De Furia, Cerami, Bunn, Lu and Peterson (Proc. natn. Acad. Sci. U.S.A., 70,$3707 ; 1973$ ) have re-examined the effect of aspirin on the oxygen affinity of haemoglobin. They find that although the acetyl group is indeed transferred, both in vitro and in vivo, there is no effect on the oxygen equilibrium of haemoglobin, either in solu tion or in intact red cells. Furthermore, patients on long-term aspirin therapy show no shift in the oxygen affinity of their blood. They also failed to observe any inhibition of in vitro sickling of deoxygenated sickle cell erythrocytes previously incubated with aspirin, and conclude that this drug is of no value in the treatment of sickle cell disease.

The gelation of sickle cell haemoglobin itself has been the subject of theoretical study. Minton ( $J$. molec. Biol., 82, 483; 1974) has devised a thermodynamic model to account for the macroscopic solution properties of $\mathrm{Hb}-\mathrm{S}$ in terms of microscopic structure and interactions. In this model step 1, the formation of a rod-like microtubular array, is treated as being equivalent to a precipitation. Step 2, the formation of the nematic gel phase, is treated as an isotropically-aligned transition in a suspension of interacting rod-like particles. By combining the two steps a qualitative temperaturecomposition phase diagram is obtained which provides a unified interpretation of many of the observed properties of $\mathrm{Hb}-\mathrm{S}$ solutions.

In $\mathrm{Hb}-\mathrm{M}$ (Boston) the distal histidines in the haem pockets of the $\alpha$ submits are replaced by tyrosines; His E7(58) $\rightarrow$ Tyr. The iron atoms of the abnormal a subunits remain in the ferric state to give a natural valency hybrid $\alpha_{2}+\mathrm{M}$ Boston $\beta_{2}$ deoxy. In heterozygotes for $\mathrm{Hb}-\mathrm{M}($ Boston) there is methaemoglobinemia because of the ferric $\propto$ chains and cyanosis because of the low oxygen affinity of the normal $\beta$ chains. Pulsinelli, Perutz and Nagel (Proc. natn. Acad. Sci. U.S.A., 70, $3870 ;$ 1973) have now studied this variant by X-ray analysis. A difference electron density map of deoxy Hb-M(Boston) minus deoxy $\mathrm{Hb}-\mathrm{A}$ shows that the ferric iron atom in the $\alpha$ subunit coordinated to the four nitrogens of the porphyrin and the phenolate group of the tyrosine. The normal bond to the proximal histidine F8 (87) is absent and the iron, which is therefore five-coordinate, lies on the distal side of the porphyrin ring (see figure). The rupture of this bond causes the $F$ helix to shorten slightly "like a spring released from a tension that had kept it slightly un-

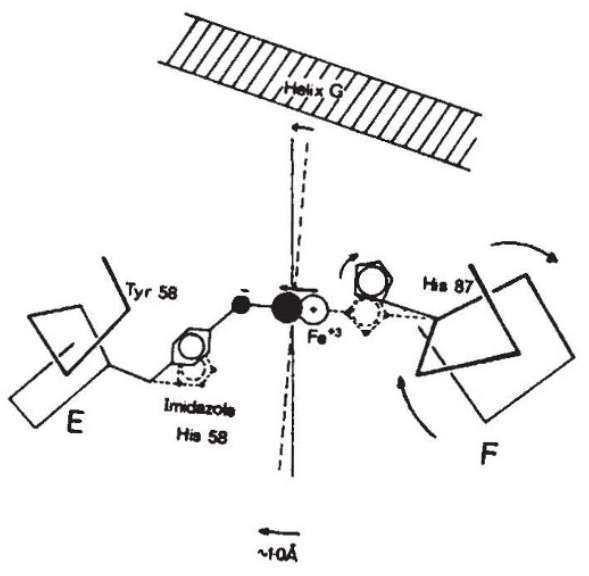

Stereochemical changes in the haem pocket of the $\alpha$ subunit on going from deoxy $\mathrm{Hb}$ to $\mathrm{Hb}-\mathrm{M}$ (Boston) (from Pulsinelli et al.).

coiled". The resulting changes in tertiary structure of the $\alpha$ subunits stabilizes the deoxy or $T$ structure of the haemoglobin tetramer, which lowers the oxygen affinity of the normal $\beta$ subunits. Thus the clinical effects and many of the abnormal properties of Hb-M(Boston) can now be accounted for in struotural terms.

Haemoglobin Little Rock is a high oxygen affinity variant which is unique in having normal Bohr effect and haem-haem interactions. Collaboration between Bare, Alben and Bromberg (Ohio), Jones and Brimhall (Oregon) and Padilla (Little Rock) (J. biol. Chem., 249, 773; 1974), has now established that the alteration in sequence is $\beta$ His $\mathrm{H} 21(143) \rightarrow$ Gln. Since the $\beta 143$ histidine is an important binding site for the allosteric effector 2,3-diphosphoglycerate, they have studied the effect of DPG on the oxygen affinity of $\mathrm{Hb}-$ Little Rock. They find that the DPG dissociation constant for deoxy Hb-Little Rock is about twice that for deoxy $\mathrm{Hb}-\mathrm{A}$, and the corresponding value for the oxy form about four times that of oxy $\mathbf{H b}-\mathbf{A}$. They conclude, however, that the higher oxygen affinity of $\mathrm{Hb}$-Little Rock cannot be explained solely in terms of reduced allosteric interaction with DPG, and that the Gln residues at $\beta$ H21(143) must have a further specific effect on the oxygenation equilibrium.

\section{Immunodepression in protozoan infection}

from our Parasitology Correspondent ANY ideas that patients with malaria or sleeping sickness readily succumb to other infections because of malnutrition associated with the disease are losing ground as more evidence accumulates to suggest that the immune response to other antigens is depressed 